\title{
A fixed-time output feedback control scheme for double integrator systems
}

DOI:

10.1016/j.automatica.2017.01.007

\section{Document Version}

Accepted author manuscript

Link to publication record in Manchester Research Explorer

\section{Citation for published version (APA):}

Tian, B., Zuo, Z., Yan, X., \& Wang, H. (2017). A fixed-time output feedback control scheme for double integrator systems. Automatica, 80, 17-24. https://doi.org/10.1016/j.automatica.2017.01.007

\section{Published in:}

Automatica

\section{Citing this paper}

Please note that where the full-text provided on Manchester Research Explorer is the Author Accepted Manuscript or Proof version this may differ from the final Published version. If citing, it is advised that you check and use the publisher's definitive version.

\section{General rights}

Copyright and moral rights for the publications made accessible in the Research Explorer are retained by the authors and/or other copyright owners and it is a condition of accessing publications that users recognise and abide by the legal requirements associated with these rights.

\section{Takedown policy}

If you believe that this document breaches copyright please refer to the University of Manchester's Takedown Procedures [http://man.ac.uk/04Y6Bo] or contact uml.scholarlycommunications@manchester.ac.uk providing relevant details, so we can investigate your claim.

\section{OPEN ACCESS}




\title{
A Fixed-time Output Feedback Control Scheme for Double Integrator Systems *
}

\author{
Bailing Tian ${ }^{\mathrm{a}, \mathrm{b}}$, Zongyu Zuo ${ }^{\mathrm{c}}$, Xiaomo Yan ${ }^{\mathrm{d}}$, Hong Wang ${ }^{\mathrm{e}}$ \\ ${ }^{a}$ School of Electrical and Information Engineering, Tianjin University, Tianjin 300072, China \\ ${ }^{\mathrm{b}}$ State Key Laboratory of Synthetical Automation for Process Industries, Northeastern University, Shenyang 110004 China \\ ${ }^{\mathrm{c}}$ The Seventh Research Division, Beihang University (BUAA), Beijing 100191, China \\ ${ }^{\mathrm{d}}$ School of Electrical and Electronic Engineering, The University of Manchester, M13 9PL, UK \\ e Pacific Northwest National Laboratory, Richland, WA 99352, USA
}

\begin{abstract}
A continuous output feedback control scheme rendering the closed-loop double integrator system globally stable in finite-time is presented. In particular, the convergence time is independent of initial conditions. The bi-limit homogeneous technique is used for controller and observer designs with fixed-time convergence. Then, a continuous output feedback control law is proposed for nominal double-integrator system and its perturbed version. The homogeneity and Lyapunov techniques are used to ensure the fixed-time stability of the closed-loop system under output feedback control framework. Finally, the efficiency of the proposed algorithms are illustrated by numerical simulations.
\end{abstract}

Key words: Output-feedback control; Fixed-time stability; Bi-homogeneity; Double integrator system

\section{Introduction}

The system with double-integrator dynamics is one of the most fundamental systems in control theory and has many applications in practice, such as spacecraft rotation [1], rotary crane motion [2] and manipulator motion [3]. Moreover, a design method developed for this system can be extended to a more general case (via backstepping, for example). Therefore, control of a doubleintegrator system has been of interest since the early days of control theory. Most of the existing control techniques provide an asymptotic or exponential stability which implies the convergence of the system trajectories to an equilibrium state over infinite horizon. In

\footnotetext{
^ This work was supported by the National Natural Science Foundation of China $(61573022,61673034,61673294,61333007)$ and The Ministry of Education Equipment Development Fund (6141A0233). This paper was not presented at any conference. Corresponding author B.L. Tian, Tel. +86-022-27892382.

Email addresses: bailing_tian@tju.edu.cn (Bailing Tian), zzybobby@buaa.edu.cn (Zongyu Zuo), xiaomo.yan@manchester.ac.uk (Xiaomo Yan), hong.wang@pnnl.gov (Hong Wang).
}

practice, finite-time convergence is much more desirable. Moreover, the closed-loop systems under finite-time control law usually demonstrate higher accuracy and better disturbance rejection properties [4]. Consequently, the finite-time control techniques have been an intensive subject in the last years.

The benchmark work on finite-time control was proposed by Bhat in [4] where the relationship between the regularity properties of Lyapunov function and the convergence time was established. Thereafter, a number of finite-time control schemes have been developed based on Lyapunov function methods [5], [6]. Another finite-time control technique is frequently associated with high-order sliding mode (HOSM) controls providing finite-time convergence to a sliding manifold. The theory of HOSM was well-developed in [7] and [8]. Many applications of the method can be found in aerospace systems [9], mechanical systems [3] and electric systems [10]. Also, the finite-time output feedback was discussed in [11] for a double-integrator system. However, the convergence time in these methods grows unboundedly when initial condition tends to infinity. Recently, the fixed-time stability theory, first developed in [12], 
has been intensively investigated in [13],[14],[15],[16] and [17]. The fixed-time stability is stronger than the finitetime stability and it means that the convergence time of a global finite-time stable system is bounded independen$\mathrm{t}$ of initial conditions. This property is especially useful for either hybrid or switching systems with some kind of dwell time. In addition, it looks promising for a system if a controller or an observer can be designed to ensure that the convergence is achieved in a fixed-time duration regardless of initial conditions. A sufficient condition for the fixed-time stability of nonlinear systems was provided in [13] and some fixed-time control laws, such as [18] and [19], were derived based on this condition. However, it is nontrivial to construct a Lyapunov function satisfying particular conditions for high-order systems. The concept of bi-limit homogeneity introduced in [12] shows that an asymptotically stable system is fixed-time stable if it is homogeneous with negative degree in 0limit, and homogeneous with positive degree in $\infty$-limit, which provides an alternate way to design a fixed-time controller or observer. An arbitrary order robust sliding mode differentiator was designed based on bi-limit homogeneous technique in [20] providing uniform convergence regardless of initial conditions. The existing works have been focused mainly on state feedback and however the output feedback is seldom discussed. It should be noted that the linear growth condition, used in the stability proof for systems under finite-time output feedback control [11], does not hold for systems under fixedtime output feedback control. Therefore, the finite-time escape may occur which makes the design of fixed-time output feedback more challenging.

Motivated by the above observations, a new fixed-time output feedback control scheme is proposed in this work. The main contributions of this paper are twofold. First, an unified framework for fixed-time convergent controller and observer is developed based on bi-limit homogeneous technique. Second, a continuous fixedtime output feedback control law is constructed and the fixed-time stability proof for the closed-loop system is derived by using the homogeneity and Lyapunov function.

The organization of the paper is as follows. Section II is devoted to some definitions and lemmas related to the homogeneity. Then, the state feedback controller and the state observer are designed in Section III and IV, respectively. In Section V, the synthesis design is introduced to stabilize the double-integrator systems by output feedback. The simulation results are provided in Section VI. Finally, the concluding remarks are summarized in Section VII.

\section{Preliminaries}

\subsection{Notations}

Throughout the paper, the following notations will be used. $\mathbb{R}$ is the set of real numbers and $\mathbb{R}_{+}=\{x \in \mathbb{R}: x>$ $0\}$. For any non-negative real number $\alpha$, the function $x \mapsto\lceil x\rfloor^{\alpha}$ is defined as $\lceil x\rfloor^{\alpha}=|x|^{\alpha} \operatorname{sign}(x)$ for any $x \in$ $\mathbb{R}$. It follows from the definition that $\frac{d\lceil x\rfloor^{\alpha}}{d x}=\alpha|x|^{\alpha-1}$, $\lceil x\rfloor^{0}=\operatorname{sign}(x),\lceil x\rfloor=x$ and $\lceil x\rfloor^{2}=x|x|$. The function $\Gamma: \mathbb{R}_{+}^{2} \mapsto \mathbb{R}$ is defined as $\Gamma(a, b)=\frac{a}{1+a}(1+b)$ for any $a, b \in \mathbb{R}_{+}$.

\subsection{Definitions and Lemmas}

Consider the nonlinear dynamical system

$$
\dot{x}(t)=f(x(t)), t>t_{0}, x\left(t_{0}\right)=x_{0}
$$

where $x=\left[x_{1}, . ., x_{n}\right]^{T} \in \mathbb{R}^{n}$ is the state vector, $f(x)$ : $\mathbb{R}^{n} \rightarrow \mathbb{R}^{n}$ is a possibly discontinuous vector field. In this case, the solutions of (1) are understood in the sense of Filippov $[21,22]$. It is assumed that the origin is an equilibrium point of system (1). Throughout the paper, it is assumed that the solution of (1) starts at $t_{0}=0$, denoted by $X\left(t, x_{0}\right)$ with $x_{0}$ as the initial condition.

Let $r=\left[r_{1}, \ldots, r_{n}\right] \in \mathbb{R}^{n}$ be the weight vector with $r_{i}>0,(i=1, \ldots, n)$. The dilation mapping is defined as $\Lambda_{\lambda}^{r}(x)=\left[\lambda^{r_{1}} x_{1}, \ldots, \lambda^{r_{n}} x_{n}\right]^{T}$ for any $\lambda>0$.

Definition 1 [23] A function $g(x): \mathbb{R}^{n} \rightarrow \mathbb{R}$ is said to be $r$-homogeneous with degree $k \in \mathbb{R}$ if for all $x \in \mathbb{R}^{n}$ and all $\lambda>0$ we have $g\left(\Lambda_{\lambda}^{r}(x)\right)=\lambda^{k} g(x)$.

Definition 2 [23] A vector field $f(x): \mathbb{R}^{n} \rightarrow \mathbb{R}^{n}$ is said to be r-homogeneous with degree $k$ if for each $i \in$ $\{1, \ldots, n\}$, the component $f_{i}(x)$ is r-homogeneous of degree of $k+r_{i}$; that is $f_{i}\left(\Lambda_{\lambda}^{r}(x)\right)=\lambda^{k+r_{i}} f_{i}(x)$ for any $\lambda>0$ and $x \in \mathbb{R}^{n}$.

Definition 3 [12] A function $g(x): \mathbb{R}^{n} \rightarrow \mathbb{R}$ is said to be homogeneous in the $p$-limit $(p=0$ or $\infty)$ with triple $\left(r_{p}, k_{p}, g_{p}\right)$, where $r_{p}=\left[r_{p, 1}, \ldots, r_{p, n}\right] \in \mathbb{R}^{n}$ is the weight vector, $k_{p}$ is the degree, and $g_{p}$ the approximating function, if $g(x)$ is continuous, $g_{p}$ is continuous and not identically zero, and, for each compact set $C \in \mathbb{R}^{n} \backslash\{0\}$, the condition $\lim _{\lambda \rightarrow p} \max _{x \in C}\left|\lambda^{-k_{p}} g\left(\Lambda_{\lambda}^{r_{p}}(x)\right)-g_{p}(x)\right|=0$ holds.

Definition 4 [12] $A$ vector field $f(x): \mathbb{R}^{n} \rightarrow \mathbb{R}^{n}$ is said to be homogeneous in the p-limit with associated triple $\left(r_{p}, k_{p}, f_{p}\right)$ where $r_{p}=\left[r_{p, 1}, \ldots, r_{p, n}\right] \in \mathbb{R}^{n}$ is the weight vector, $k_{p}$ is the degree and $f_{p}$ the approximating vector field, if, for each $i, k_{p}+r_{p, i}>0$ and the function $f_{i}(x)$ is homogeneous in the $p$-limit with associated triple $\left(r_{p}, k_{p}+r_{p, i}, f_{p, i}\right)$. 
Definition 5 [12] A function (resp. vector field) is said to be homogeneous in the bi-limit if it is homogeneous in the 0-limit and homogeneous in the $\infty$-limit simultaneously.

Definition 6 [7] The origin of system (1) is said to be globally finite-time stable if it is Lyapunov stable and for any $R>0$ exists $T>0$, such that any trajectory starting within the ball $\|x\| \leq R$ stabilizes at the origin in the time $T$.

Definition 7 [13] The origin of system (1) is said to be fixed-time stable if it is globally finite-time stable and there exists a fixed positive constant $T_{\max }$ such that $T \leq$ $T_{\max }$ for any $x_{0} \in \mathbb{R}^{n}$.

Remark 8 From the definitions, it can be seen that the convergence time $T$ for fixed-time stable system$s$ is bounded even when initial condition $x_{0}$ tend$s$ to infinity. To illustrate this point, the following two examples are considered. The origin of system $\dot{x}=-\lceil x\rfloor^{\frac{1}{2}}, x(0)=x_{0}, x \in \mathbb{R}$ is finite-time stable, since its solution has the form $X\left(t, x_{0}\right)=$ $\operatorname{sign}\left(x_{0}\right)\left[\sqrt{\left|x_{0}\right|}-\frac{1}{2} t\right]^{2}$ for $t<2 \sqrt{\left|x_{0}\right|}$ and $X\left(t, x_{0}\right)=0$ for $t \geq 2 \sqrt{\left|x_{0}\right|}$. The convergence time $T=2 \sqrt{\left|x_{0}\right|} \rightarrow \infty$ as $\left|x_{0}\right| \rightarrow \infty$. The origin of system $\dot{x}=-\lceil x\rfloor^{\frac{1}{2}}-\lceil x\rfloor^{2}$ is fixed-time stable, since its solution is denoted by $X\left(t, x_{0}\right)=\operatorname{sign}\left(x_{0}\right) \tan ^{2}\left(\arctan \left(\sqrt{\left|x_{0}\right|}\right)-0.5 t\right)$ for $t<2 \arctan \left(\sqrt{\left|x_{0}\right|}\right)$ and $X\left(t, x_{0}\right)=0$ for $t \geq$ $2 \arctan \left(\sqrt{\left|x_{0}\right|}\right)$. The convergence time $T$ is globally bounded satisfying $T \leq 2 \arctan \left(\sqrt{\left|x_{0}\right|}\right) \leq \pi$.

Definition 9 The origin of system (1) is said to be practically fixed-time stable, if it is Lyapunov stable and there exist a bounded region $\Omega \in \mathbb{R}^{n}$ and $T>0$ such that for any $R>0$ any trajectory starting within the ball $\|x\| \leq R$ converges to $\Omega$ in the time $T$ and stays in $\Omega$ forever.

Lemma 10 [12] For system (1), suppose that $f(x)$ is a homogeneous in the bi-limit with associated triples $\left(r_{0}, k_{0}, f_{0}\right)$ and $\left(r_{\infty}, k_{\infty}, f_{\infty}\right)$. If the origins of systems $\dot{x}=f(x), \dot{x}_{0}=f_{0}(x)$ and $\dot{x}_{\infty}=f_{\infty}(x)$ are globally asymptotically stable, then the following statements hold

1) The origin of (1) is fixed-time stable when condition $k_{\infty}>0>k_{0}$ holds;

2) Let $d_{V_{0}}$ and $d_{V_{\infty}}$ be real numbers such that $d_{V_{0}}>$ $\max _{1 \leq i \leq n} r_{0, i}$ and $d_{V_{\infty}}>\max _{1 \leq i \leq n} r_{\infty, i}$. There exists a continuous, positive definite and proper function $V(x)$ such that the function $\frac{\partial V}{\partial x_{i}}$ is homogeneous in the bi-limit with triples $\left(r_{0}, d_{V_{0}}-r_{0, i}, \frac{\partial V_{0}}{\partial x_{i}}\right)$ and $\left(r_{\infty}, d_{V_{\infty}}-r_{\infty, i}, \frac{\partial V_{\infty}}{\partial x_{i}}\right)$ and the function $\frac{\partial V}{\partial x} f(x)$ is negative definite.

\section{$3 \quad$ Fixed-Time State Feedback Control}

To begin with, we consider the fixed-time stability of the following double-integrator system

$$
\dot{x}_{1}=x_{2}, \dot{x}_{2}=u, x(0)=x_{0}
$$

where $x_{1}$ and $x_{2} \in \mathbb{R}$ are the measurable state variables and $u \in \mathbb{R}$ is the control input. To ensure the fixedtime stability of system (2), the following control law is proposed

$$
\begin{aligned}
u(x) & =-\left(k_{1}\left\lceil x_{1}\right\rfloor^{\varrho_{1}}+k_{1}^{\prime}\left\lceil x_{1}\right\rfloor+k_{1}^{\prime \prime}\left\lceil x_{1}\right\rfloor^{\varrho_{1}^{\prime}}\right)-\left(k_{2}\left\lceil x_{2}\right\rfloor \varrho_{2}\right. \\
& \left.+k_{2}^{\prime}\left\lceil x_{2}\right\rfloor+k_{2}^{\prime \prime}\left\lceil x_{2}\right\rfloor_{2}^{\varrho_{2}^{\prime}}\right)
\end{aligned}
$$

where parameters $k_{i}>0, k_{i}^{\prime}>0, k_{i}^{\prime \prime}>0,(i=1,2)$ and $\varrho_{i}, \varrho_{i}^{\prime},(i=1,2)$ are given by

$$
\varrho_{1}=\frac{\varrho}{2-\varrho}, \varrho_{2}=\varrho, \varrho_{1}^{\prime}=\frac{4-3 \varrho}{2-\varrho}, \varrho_{2}^{\prime}=\frac{4-3 \varrho}{3-2 \varrho}
$$

with $\varrho \in(0,1)$. The parameters provided in (4) are chosen from the homogeneity reasoning. To show that, let $f_{s}(x)=\left[x_{2}, u(x)\right]^{T}$ denote the vector field of closed-loop system (2) under control law (3). Furthermore, define vectors

$$
\begin{gathered}
f_{s_{0}}(x)=\left[x_{2},-k_{1}\left\lceil x_{1}\right\rfloor^{\varrho_{1}}-k_{2}\left\lceil x_{2}\right\rfloor^{\varrho_{2}}\right]^{T} \\
f_{s_{\infty}}(x)=\left[x_{2},-k_{1}^{\prime \prime}\left\lceil x_{1}\right\rfloor_{1}^{\varrho_{1}^{\prime}}-k_{2}^{\prime \prime}\left\lceil x_{2} \varrho^{\varrho_{2}^{\prime}}\right]^{T}\right.
\end{gathered}
$$

It can be observed from (4) that $0<\varrho_{i}<1<\varrho_{i}^{\prime}$, $(i=$ $1,2)$ hold for any $\varrho \in(0,1)$. It follows that $f_{s_{0}}(x)$ and $f_{s_{\infty}}(x)$ can be considered as approximating functions for $f_{s}(x)$ in 0 -limit and $\infty$-limit, respectively. Furthermore, a direct verification shows, taking $r_{s_{0}}=\left[\frac{2-\varrho}{1-\varrho}, \frac{1}{1-\varrho}\right]^{T}$, the vector field $f_{s_{0}}(x)$ is $r_{s_{0}}$-homogeneous of degree $k_{s_{0}}=-1$. Similarly, the vector field $f_{s_{\infty}}(x)$ is $r_{s_{\infty}}$-homogeneous of degree $k_{s_{\infty}}=1$ when fixing $r_{s_{\infty}}=\left[\frac{2-\varrho}{1-\varrho}, \frac{3-2 \varrho}{1-\varrho}\right]^{T}$. Therefore, the closed-loop system (2) under the control law (3) with parameters (4) is homogeneous in the bi-limit with $\left(r_{s_{0}}, k_{s_{0}}, f_{s_{0}}(x)\right)$ and $\left(r_{s_{\infty}}, k_{s_{\infty}}, f_{s_{\infty}}(x)\right)$.

Theorem 11 Consider double-integrator system (2) under control law (3) with parameters given in (4). Then, the origin of system (2) is fixed-time stable if the parameters $k_{i}>0, k_{i}^{\prime}>0, k_{i}^{\prime \prime}>0,(i=1,2)$ and $\varrho \in(0,1)$ are selected.

Proof. The proof is divided into two steps. First, we prove that the closed-loop system (2) under the control law (3) with the parameters provided in (4) is globally asymptotically stable. Then, we show that its approximating systems in 0 -limit and $\infty$-limit are also globally 
asymptotically stable. Taking into account the bi-limit homogeneity of the system, it follows from Lemma 10 that the proof is completed.

To examine the stability of this system, consider the Lyapunov function candidate:

$$
\begin{aligned}
V & =2 k_{1}\left(\varrho_{1}^{\prime}+1\right)\left|x_{1}\right|^{\varrho_{1}+1}+k_{1}^{\prime}\left(\varrho_{1}+1\right)\left(\varrho_{1}^{\prime}+1\right)\left|x_{1}\right|^{2} \\
& +2 k_{1}^{\prime \prime}\left(\varrho_{1}+1\right)\left|x_{1}\right|^{\varrho_{1}^{\prime}+1}+\left(\varrho_{1}+1\right)\left(\varrho_{1}^{\prime}+1\right)\left|x_{2}\right|^{2}
\end{aligned}
$$

It is obvious that $V$ in (7) is continuously differentiable, positive definite and radially unbounded. The derivative of $V$ along the trajectories in (2) with control (3) is derived by

$$
\dot{V}=k_{1} \Pi\left\lceil x_{1}\right\rfloor^{\varrho_{1}} \dot{x}_{1}+k_{1}^{\prime} \Pi\left\lceil x_{1}\right\rfloor \dot{x}_{1}+k_{1}^{\prime \prime} \Pi\left\lceil x_{1}\right\rfloor^{\varrho_{1}^{\prime}} \dot{x}_{1}+\Pi\left\lceil x_{2}\right\rfloor \dot{x}_{2}
$$

where $\Pi=2\left(\varrho_{1}+1\right)\left(\varrho_{1}^{\prime}+1\right)$. Substituting $(2)$ and $(3)$ into (8) results in

$$
\begin{aligned}
\dot{V} & =\Pi x_{2}\left(k_{1}\left\lceil x_{1}\right\rfloor^{\varrho_{1}}+k_{1}^{\prime}\left\lceil x_{1}\right\rfloor+k_{1}^{\prime \prime}\left\lceil x_{1}\right\rfloor^{\varrho_{1}^{\prime}}\right)-\Pi\left\lceil x_{2}\right\rfloor \\
& \times\left(k_{1}\left\lceil x_{1}\right\rfloor^{\varrho_{1}}+k_{1}^{\prime}\left\lceil x_{1}\right\rfloor+k_{1}^{\prime \prime}\left\lceil x_{1} \varrho_{1}^{\prime}+k_{2}\left\lceil x_{2}\right\rfloor^{\varrho_{2}}\right.\right. \\
& \left.+k_{2}^{\prime}\left\lceil x_{2}\right\rfloor+k_{2}^{\prime \prime}\left\lceil x_{2}\right\rfloor \varrho_{2}^{\prime}\right)
\end{aligned}
$$

Since $\left\lceil x_{2}\right\rfloor=x_{2}$ and $\left\lceil x_{2}\right\rfloor\left\lceil x_{2}\right\rfloor^{\alpha}=\left|x_{2}\right|\left|x_{2}\right|^{\alpha}$ hold for any $\alpha \geq 0,(9)$ can be rewritten as

$$
\dot{V}=-\Pi\left|x_{2}\right|\left(k_{2}\left|x_{2}\right|^{\varrho_{2}}+k_{2}^{\prime}\left|x_{2}\right|+k_{2}^{\prime \prime}\left|x_{2}\right|^{\varrho_{2}^{\prime}}\right)
$$

If follows from (10) that $\dot{V}<0$ everywhere except on the line $x_{2}=0$ where $\dot{V}=0$. Furthermore, note that for system (2) under control law (3) to guarantee the condition $\dot{V}=0$, the system trajectory must evolve on $x_{1}=x_{2}=0$. By applying LaSalle invariance principle [25], we have that the origin of the closed-loop system is globally asymptotically stable.

For the system $\dot{x}=f_{s_{0}}(x)$ with $f_{s_{0}}(x)$ being defined in (5), if the Lyapunov function is chosen as $V_{0}=0.5\left(\varrho_{1}+1\right)\left|x_{2}\right|^{2}+k_{1}\left|x_{1}\right|^{\varrho_{1}+1}$, it can be easily calculated that $\dot{V}_{0}=-\left(\varrho_{1}+1\right) k_{2}\left|x_{2}\right|^{\varrho_{2}+1}$. Similarly, if we choose $V_{\infty}=0.5\left(\varrho_{1}^{\prime}+1\right)\left|x_{2}\right|^{2}+k_{1}^{\prime \prime}\left|x_{1}\right|_{1}^{\prime}+1$ for system $\dot{x}=f_{s_{\infty}}(x)$ with $f_{s_{\infty}}(x)$ being defined in (6), it can be seen that the equality $\dot{V}_{\infty}=-\left(\varrho_{1}^{\prime}+1\right) k_{2}^{\prime \prime}\left|x_{2}\right|^{\varrho_{2}^{\prime}+1}$ holds for any $x \in \mathbb{R}^{2}$. The LaSalle invariance principle [25] ensures the global asymptotic stability of systems $\dot{x}=f_{s_{0}}(x)$ and $\dot{x}=f_{s_{\infty}}(x)$.

It follows from the above analysis that all the conditions in the first statement of Lemma 10 are satisfied, which, in turn, prove the conclusion. This completes the proof.

\section{Fixed-Time State Observer}

In this section, a new fixed-time state observer is developed for the double-integrator system as follows

$$
\left\{\begin{array}{l}
\dot{\hat{x}}_{1}=\hat{x}_{2}+\bar{k}_{1}\left\lceil e_{1}\right\rfloor^{\bar{\varrho}_{1}}+\bar{k}_{1}^{\prime}\left\lceil e_{1}\right\rfloor+\bar{k}_{1}^{\prime \prime}\left\lceil e_{1}\right\rfloor \bar{\varrho}_{1}^{\prime} \\
\dot{\hat{x}}_{2}=u+\bar{k}_{2}\left\lceil e_{1}\right\rfloor^{\bar{\varrho}_{2}}+\bar{k}_{2}^{\prime}\left\lceil e_{1}\right\rfloor+\bar{k}_{2}^{\prime \prime}\left\lceil e_{1}\right\rfloor^{\bar{\varrho}_{2}^{\prime}}
\end{array}\right.
$$

with $e_{1}=x_{1}-\hat{x}_{1}, \bar{k}_{i}>0, \bar{k}_{i}^{\prime}>0, \bar{k}_{i}^{\prime \prime}>0,(i=1,2)$ and the parameters $\bar{\varrho}_{i}, \bar{\varrho}_{i}^{\prime},(i=1,2)$ are given by

$$
\bar{\varrho}_{1}=\bar{\varrho}, \bar{\varrho}_{2}=2 \bar{\varrho}-1, \bar{\varrho}_{1}^{\prime}=2-\bar{\varrho}, \bar{\varrho}_{2}^{\prime}=3-2 \bar{\varrho}
$$

where $\bar{\varrho} \in\left(\frac{1}{2}, 1\right)$. Subtracting (11) from (2) yields the following error dynamics

$$
\left\{\begin{array}{l}
\dot{e}_{1}=-\bar{k}_{1}\left\lceil e_{1}\right\rfloor^{\bar{\varrho}_{1}}-\bar{k}_{1}^{\prime}\left\lceil e_{1}\right\rfloor-\bar{k}_{1}^{\prime \prime}\left\lceil e_{1}\right\rfloor^{\bar{\varrho}_{1}^{\prime}}+e_{2} \\
\dot{e}_{2}=-\bar{k}_{2}\left\lceil e_{1}\right\rfloor^{\bar{\varrho}_{2}}-\bar{k}_{2}^{\prime}\left\lceil e_{1}\right\rfloor-\bar{k}_{2}^{\prime \prime}\left\lceil e_{1}\right\rfloor \bar{\varrho}_{2}^{\prime}
\end{array}\right.
$$

with $e_{2}=x_{2}-\hat{x}_{2}$. The parameters (12) are designed to ensure that the system (13) is bi-limit homogeneous. To illustrate that, define $e=\left[e_{1}, e_{2}\right]^{T}$ and let $f_{p}(e)$ be the vector field of system (13). Then, system (13) can be rewritten as $\dot{e}=f_{p}(e)$. With the following definitions

$$
\begin{gathered}
f_{p_{0}}(e)=\left[-\bar{k}_{1}\left\lceil e_{1}\right\rfloor^{\bar{\varrho}_{1}}+e_{2},-\bar{k}_{2}\left\lceil e_{1}\right\rfloor^{\bar{\varrho}_{2}}\right]^{T}, \\
f_{p_{\infty}}(e)=\left[-\bar{k}_{1}^{\prime \prime}\left\lceil e_{1}\right\rfloor^{\bar{\varrho}_{1}^{\prime}}+e_{2},-\bar{k}_{2}^{\prime \prime}\left\lceil e_{1}\right\rfloor^{\bar{\varrho}_{2}^{\prime}}\right]^{T}
\end{gathered}
$$

it can be found that $f_{p_{0}}(e)$ is homogeneous with degree $k_{p_{0}}=-1$ with respect to the weight vector $r_{p_{0}}=\left[\frac{1}{1-\bar{\varrho}}, \frac{\bar{\varrho}}{1-\bar{\varrho}}\right]^{T}$ and $f_{p_{\infty}}(e)$ is homogeneous with degree $k_{p_{\infty}}=1$ with respect to the weight vector $r_{p_{\infty}}=\left[\frac{1}{1-\bar{\varrho}}, \frac{2-\bar{\varrho}}{1-\bar{\varrho}}\right]^{T}$. Since $\bar{\varrho} \in\left(\frac{1}{2}, 1\right)$, it can be observed from (12) that conditions $0<\bar{\varrho}_{i}<1<\bar{\varrho}_{i}^{\prime},(i=1,2)$ hold. Therefore, the vector fields $f_{p_{0}}(e)$ and $f_{p_{\infty}}(e)$ can be referred to as approximating homogeneous functions for $f_{p}(e)$ in 0 -limit and $\infty$-limit, respectively.

The main result in this section is summarized in the following theorem.

Theorem 12 Consider the observer (11) with parameters defined in (12). Then, the resulting error dynamics given in (13) are fixed-time stable if the parameters $\bar{\varrho} \in\left(\frac{1}{2}, 1\right)$ and $\bar{k}_{i}>0, \bar{k}_{i}^{\prime}>0, \bar{k}_{i}^{\prime \prime}>0,(i=1,2)$ are selected.

Proof. Consider a Lyapunov function candidate

$$
\begin{aligned}
V & =\bar{k}_{2}(2-\bar{\varrho})\left|e_{1}\right|^{2 \bar{\varrho}}+\bar{k}_{2}^{\prime \prime} \bar{\varrho}\left|e_{1}\right|^{4-2 \bar{\varrho}}+\bar{k}_{2}^{\prime} \bar{\varrho}(2-\bar{\varrho})\left|e_{1}\right|^{2} \\
& +\bar{\varrho}(2-\bar{\varrho})\left|e_{2}\right|^{2}
\end{aligned}
$$

It is obvious that $V$ in (16) is continuously differentiable, positive definite and radially unbounded for any $\bar{\varrho} \in$ 
$\left(\frac{1}{2}, 1\right)$. The time derivative of Lyapunov function is given by

$\dot{V}=\Xi\left(\bar{k}_{2}\left\lceil e_{1}\right\rfloor^{(2 \rho-1)}+\bar{k}_{2}^{\prime \prime}\left\lceil e_{1}\right\rfloor^{(3-2 \rho)}+\bar{k}_{2}^{\prime}\left\lceil e_{1}\right\rfloor\right) \dot{e}_{1}+\Xi\left\lceil e_{2}\right\rfloor \dot{e}_{2}$

with $\Xi=2 \bar{\varrho}(2-\bar{\varrho})$. Substituting (13) into (17) results in

$$
\begin{aligned}
\dot{V} & =-\Xi \bar{k}_{2}\left[\bar{k}_{1}\left|e_{1}\right|^{3 \bar{\varrho}-1}+\bar{k}_{1}^{\prime}\left|e_{1}\right|^{2 \bar{\varrho}}+\bar{k}_{1}^{\prime \prime}\left|e_{1}\right|^{\bar{\varrho}+1}\right] \\
& -\Xi \bar{k}_{2}^{\prime \prime}\left[\bar{k}_{1}\left|e_{1}\right|^{3-\varrho}+\bar{k}_{1}^{\prime}\left|e_{1}\right|^{4-2 \varrho}+\bar{k}_{1}^{\prime \prime}\left|e_{1}\right|^{5-3 \varrho}\right] \\
& -\Xi\left[\bar{k}_{1}\left|e_{1}\right|^{2 \bar{\varrho}}+\bar{k}_{1}^{\prime}\left|e_{1}\right|^{2}+\bar{k}_{1}^{\prime \prime}\left|e_{1}\right|^{3-\varrho}\right]
\end{aligned}
$$

It follows that $\dot{V} \leq 0$ holds for any $\bar{k}_{i}>0, \bar{k}_{i}^{\prime}>0, \bar{k}_{i}^{\prime \prime}>$ $0,(i=1,2)$. Furthermore, it can be easily found that the only invariant set for system (13) is $e_{1}=e_{2}=0$ when $\dot{V}=0$. It follows from the LaSalle invariance principle that the error dynamics (13) are globally asymptotically stable.

If specified with $\bar{k}_{1}^{\prime}=\bar{k}_{2}^{\prime}=\bar{k}_{1}^{\prime \prime}=\bar{k}_{2}^{\prime \prime}=0$ (or $\bar{k}_{1}=\bar{k}_{2}=\bar{k}_{1}^{\prime}=\bar{k}_{2}^{\prime}=0$ ) in (13), (16)-(18), the global asymptotic stability of system $\dot{e}=f_{p_{0}}(e)$ (or $\left.\dot{e}=f_{p_{\infty}}(e)\right)$ can be obtained, where $f_{p_{0}}(e)\left(\right.$ or $\left.f_{p_{\infty}}(e)\right)$ is given in (14) (or (15))

Taking into account the bi-limit homogeneity of the system (13), it follows from Lemma 10 that the system (13) is fixed-time stable. This completes the proof.

\section{$5 \quad$ Fixed-Time Output Feedback Synthesis}

\subsection{Synthesis Design}

Now, we are in a position to construct a global output feedback fixed-time stabilizing control law for doubleintegrator systems. To this end, substituting $x_{2}$ in (3) by its estimated value $\hat{x}_{2}$ in (11) yields

$$
\begin{aligned}
u(\hat{x}) & =-\left(k_{1}\left\lceil x_{1}\right\rfloor^{\varrho_{1}}+k_{1}^{\prime}\left\lceil x_{1}\right\rfloor+k_{1}^{\prime \prime}\left\lceil x_{1}\right\rfloor^{\varrho_{1}^{\prime}}\right)-\left(k_{2}\left\lceil\hat{x}_{2}\right\rfloor \varrho_{2}\right. \\
& \left.+k_{2}^{\prime}\left\lceil\hat{x}_{2}\right\rfloor+k_{2}^{\prime \prime}\left\lceil\hat{x}_{2}\right\rfloor^{\varrho_{2}^{\prime}}\right)
\end{aligned}
$$

where only $x_{1}$ is measurable and all control parameters are the same with those in Theorem 11. The main results about the output feedback control is summarized in the following theorem.

Theorem 13 Consider system (2) under the output feedback control law (19) with $\hat{x}_{2}$ generated by observer (11). Then the closed-loop system (2), (11) and (19) is fixed-time stable if the controller parameters $\underline{k}_{i}, \underline{k}_{i}^{\prime}, k_{i}^{\prime \prime}, \varrho_{i}, \varrho_{i}^{\prime}(i=1,2)$ and the observer parameters $\bar{k}_{j}, \bar{k}_{j}^{\prime}, \bar{k}_{j}^{\prime \prime}, \bar{\varrho}_{j}, \bar{\varrho}_{j}^{\prime}(j=1,2)$ are selected as in Theorems 11 and 12 , respectively.
Proof. It follows form Theorem 12 that there exists a finite time, i.e., $T_{1}$, uniformly in initial estimation error $e_{1}(0)$ and $e_{2}(0)$ such that $\hat{x}_{2}(t)=x_{2}(t)$ for $t \geq T_{1}$. As a result, the output feedback control (19) coincides with state feedback control law (3) for all $t \geq T_{1}$. Furthermore, if the system trajectory under the output feedback control law does not escape during the interval $t \in\left[0, T_{1}\right]$, it follows from Theorem 11 that there exists a finite time, i.e., $T_{2}$, uniformly in $x\left(T_{1}\right)$ to ensure the fixed-time stability of double-integrator system. Therefore, the condition that the closed-loop system under output feedback control law (19) does not escape in finite time is sufficient to derive the conclusion of Theorem 13. It should be noted that the method in [11] can not be applied here, since the right hand side of the closed-loop system under (2), (11) and (19) does not satisfy the linear growth condition. To complete the proof, let us consider the Lyapunov function $V$ in (7). The derivative of $V$ along the trajectory (2) under output feedback control law (19) is given by

$$
\dot{V}=-\Pi\left(k_{2} x_{2}\left\lceil\hat{x}_{2}\right\rfloor^{\varrho_{2}}+k_{2}^{\prime} x_{2}\left\lceil\hat{x}_{2}\right\rfloor+k_{2}^{\prime \prime} x_{2}\left\lceil\hat{x}_{2}\right\rfloor_{2}^{\varrho_{2}^{\prime}}\right)
$$

with $\Pi=2\left(\varrho_{1}+1\right)\left(\varrho_{1}^{\prime}+1\right)$. Since $\hat{x}_{2}=x_{2}-e_{2}$, it follows that $x_{2}\left\lceil\hat{x}_{2}\right\rfloor^{\vartheta}=x_{2}\left|x_{2}-e_{2}\right|^{\vartheta} \operatorname{sign}\left(x_{2}-e_{2}\right)$ for any $\vartheta>0$. For the convenience of the proof, two different cases are discussed.

Case 1: It is assumed that the condition $\left|x_{2}\right|>\left|e_{2}\right|$ holds, which implies $\operatorname{sign}\left(x_{2}-e_{2}\right)=\operatorname{sign}\left(x_{2}\right)$. Therefore, one has $x_{2}\left\lceil\hat{x}_{2}\right\rfloor^{\vartheta}=\left|x_{2}\right|\left|x_{2}-e_{2}\right|^{\vartheta}$ for any $\vartheta>0$. Then, $\dot{V}$ in (20) can be rewritten as

$\dot{V}=-\Pi\left|x_{2}\right|\left(k_{2}\left|x_{2}-e_{2}\right|^{\varrho_{2}}+k_{2}^{\prime}\left|x_{2}-e_{2}\right|+k_{2}^{\prime \prime}\left|x_{2}-e_{2}\right|^{\varrho_{2}^{\prime}}\right)$

Case 2: It is assumed that the condition $\left|x_{2}\right| \leq\left|e_{2}\right|$ holds. Since $-x_{2}\left\lceil\hat{x}_{2}\right\rfloor^{\vartheta} \leq\left|x_{2}\right|\left|x_{2}-e_{2}\right|^{\vartheta}$ holds for any $\vartheta>0$ and $x_{2}, e_{2} \in R$, the $\dot{V}$ in (20) satisfies the following inequality

$$
\dot{V} \leq \Pi\left|x_{2}\right|\left(k_{2}\left|x_{2}-e_{2}\right|^{\varrho_{2}}+k_{2}^{\prime}\left|x_{2}-e_{2}\right|+k_{2}^{\prime \prime}\left|x_{2}-e_{2}\right|^{\varrho_{2}^{\prime}}\right)
$$

Taking into account $\left|x_{2}\right| \leq\left|e_{2}\right|$ and the well-known inequality $|a-b|^{\vartheta} \leq(|a|+|b|)^{\vartheta}$ for any $a, b \in \mathbb{R}$ and $\vartheta \geq 0$, the inequality (22) satisfies

$$
\dot{V} \leq \Pi\left(k_{2} 2^{\varrho_{2}}\left|e_{2}\right|^{\varrho_{2}+1}+2 k_{2}^{\prime}\left|e_{2}\right|^{2}+k_{2}^{\prime \prime} 2^{\varrho_{2}^{\prime}}\left|e_{2}\right|^{\varrho_{2}^{\prime}+1}\right)
$$

Then, we will show that there exists a positive constant $M$ such that the derivative of the Lyapunov function in (20) satisfies $\dot{V} \leq M$ at any instant of time in the both cases. Since the Theorem 12 ensures the fixed-time convergence of $e_{2}$, which implies the boundness of $e_{2}$, it 
follows that there exists a least upper bound of the right hand side of (23). Denote the least upper bound by $M=\sup \left\{\Pi\left(k_{2} 2^{\varrho_{2}}\left|e_{2}\right|^{\varrho_{2}+1}+2 k_{2}^{\prime}\left|e_{2}\right|^{2}+k_{2}^{\prime \prime} 2^{\varrho_{2}^{\prime}}\left|e_{2}\right|^{\varrho_{2}^{\prime}+1}\right)\right\}$, it follows from (21) and (23) that the $\dot{V}$ in (20) satisfies $\dot{V} \leq M$. Therefore, $V$ as well as the system states $x_{1}, x_{2}$ can not escape in any finite time interval.

From the above analysis, it can be concluded that the double-integrator system with output feedback control law (19) does not escape in any finite time interval. Following the analysis at the beginning of the proof, one has that the closed-loop system under (2), (11) and (19) is fixed-time stable. This completes the proof.

\subsection{Discussion on Robustness}

For a further discussion, a key lemma is recalled.

Lemma 14 [12] Consider system $\dot{x}=f(x, \delta)$ with exogenous disturbance vector $\delta=\left[\delta_{1}, \ldots, \delta_{m}\right]^{T} \in \mathbb{R}^{m}$ and suppose that $f(x, \delta)$ is a continuous homogeneous vector field in the bi-limit with triples $\left(\left(r_{0}, \tau_{0}\right), k_{0}, f_{0}\right)$ and $\left(\left(r_{\infty}, \tau_{\infty}\right), k_{\infty}, f_{\infty}\right)$, where $\tau_{0} \in \mathbb{R}^{m}$ and $\tau_{\infty} \in \mathbb{R}^{m}$ are weight vectors with respect to disturbance $\delta$. Then, under the hypotheses of Lemma 10, the Lyapunov function $V(x)$ given in Lemma 10 satisfies

$$
\begin{aligned}
\frac{\partial V}{\partial x} f(x, \delta) & \leq-k_{V} \Gamma\left(V^{\frac{d_{V_{0}}+k_{0}}{d_{V_{0}}}}, V^{\frac{d_{V_{\infty}}+k_{\infty}}{{ }^{V_{\infty}}}}\right) \\
& +k_{\delta} \sum_{j=1}^{m} \Gamma\left(\left|\delta_{j}\right|^{\frac{d_{V_{0}}+k_{0}}{\tau_{0, j}}},\left|\delta_{j}\right|^{\frac{d_{V_{\infty}}+k_{\infty}}{\tau_{\infty, j}}}\right)
\end{aligned}
$$

where $k_{V}$ and $k_{\delta}$ are positive real numbers and function $\Gamma$ is defined in Section II.A.

Now, we proceed to discuss the robustness of the proposed control scheme for the following perturbed doubleintegrator system

$$
\dot{x}_{1}=x_{2}, \dot{x}_{2}=u+\Delta(t), x(0)=x_{0}
$$

Assumption 15 Suppose that the perturbation $\Delta(t)$ in (25) is uniformly bounded with a known constant $\delta_{u}$, i.e., $|\Delta(t)| \leq \delta_{u}$ and also Lipschitz continuous with a Lipschitz constant $L$, i.e., $|\dot{\Delta}(t)| \leq L$.

Applying the observer (11) for system (25) yields the following error dynamics

$$
\left\{\begin{array}{l}
\dot{e}_{1}=-\bar{k}_{1}\left\lceil e_{1}\right\rfloor^{\bar{\varrho}_{1}}-\bar{k}_{1}^{\prime}\left\lceil e_{1}\right\rfloor-\bar{k}_{1}^{\prime \prime}\left\lceil e_{1}\right\rfloor^{\bar{\varrho}_{1}^{\prime}}+e_{2} \\
\dot{e}_{2}=-\bar{k}_{2}\left\lceil e_{1}\right\rfloor^{\bar{\varrho}_{2}}-\bar{k}_{2}^{\prime}\left\lceil e_{1}\right\rfloor-\bar{k}_{2}^{\prime \prime}\left\lceil e_{1}\right\rfloor^{\bar{\varrho}_{2}^{\prime}}+\Delta(t)
\end{array}\right.
$$

In order to ensure the robustness against the nonvanishing perturbation $\Delta(t)$, we select

$$
\bar{\varrho}=\frac{1}{2}, \bar{k}_{1}^{\prime}=0, \bar{k}_{1}^{\prime \prime}=\bar{k}_{1}, \bar{k}_{2}^{\prime}=\frac{4}{3} \bar{k}_{2}^{\prime \prime}=4 \bar{k}_{2}
$$

Taking into account (12), (26) can be rewritten as

$$
\left\{\begin{array}{l}
\dot{e}_{1}=-\bar{k}_{1}\left(\left\lceil e_{1}\right\rfloor^{\frac{1}{2}}+\left\lceil e_{1}\right\rfloor^{\frac{3}{2}}\right)+e_{2} \\
\dot{e}_{2}=-2 \bar{k}_{2}\left(\frac{1}{2}\left\lceil e_{1}\right\rfloor^{0}+2\left\lceil e_{1}\right\rfloor+\frac{3}{2}\left\lceil e_{1}\right\rfloor^{2}\right)+\Delta(t)
\end{array}\right.
$$

which coincides with the uniform robust exact differentiator proposed in [15]. Following the result in [15], the parameters $\bar{k}_{1}$ and $\bar{k}_{2}$ can be chosen from the following set

$$
\begin{aligned}
\Omega & =\left\{\left(\bar{k}_{1}, \bar{k}_{2}\right) \in \mathbb{R}^{2} \mid 0<\bar{k}_{1} \leq 2 \sqrt{\delta_{u}}, \bar{k}_{2}>\frac{\bar{k}_{1}^{2}}{8}+\frac{2 \delta_{u}^{2}}{\bar{k}_{1}^{2}}\right\} \\
& \cup\left\{\left(\bar{k}_{1}, \bar{k}_{2}\right) \in \mathbb{R}^{2} \mid \bar{k}_{1}>2 \sqrt{\delta_{u}}, \bar{k}_{2}>\delta_{u}\right\}
\end{aligned}
$$

in order to guarantee the fixed-time stability of system (28). Then, the perturbation $\Delta(t)$ can be approximated through equivalent control method [33]. It follows from the above analysis that there exists a finite time, i.e., $T_{1}^{\prime}$, such that $e_{1}(t)=e_{2}(t)=0$ for all $t \geq T_{1}^{\prime}$, implying the following identity holds

$$
-2 \bar{k}_{2}\left(\frac{1}{2}\left\lceil e_{1}\right\rfloor^{0}+2\left\lceil e_{1}\right\rfloor+\frac{3}{2}\left\lceil e_{1}\right\rfloor^{2}\right)+\Delta(t)=0, t \geq T_{1}^{\prime}
$$

Since $e_{1}(t)=e_{2}(t)=0$ for $t \geq T_{1}^{\prime}$, it follows that $\left\lceil e_{1}\right\rfloor=\left\lceil e_{1}\right\rfloor^{2}=0$ for $t \geq T_{1}^{\prime}$. Taking into account $\left[e_{1}\right\rfloor^{0}=\operatorname{sign}\left(e_{1}\right)$, the equivalent output injection $\Delta_{e q}(t)$ can be obtained from (30) according to the definition of equivalent control in [33]

$$
\Delta_{e q}(t)=\left.\left[\bar{k}_{2} \operatorname{sign}\left(e_{1}\right)\right]\right|_{e q}(t)=\Delta(t)
$$

with $\left.\left[\bar{k}_{2} \operatorname{sign}\left(e_{1}\right)\right]\right|_{e q}(t)$ being an average value of the discontinuous function $\bar{k}_{2} \operatorname{sign}\left(e_{1}\right)$. In practice, $\Delta_{e q}(t)$ can be approximated by $\bar{\Delta}_{e q}(t)$ using the following low pass filter

$$
\dot{\bar{\Delta}}_{e q}(t)=\frac{1}{\tau}\left[\bar{k}_{2} \operatorname{sign}\left(e_{1}\right)-\bar{\Delta}_{e q}\right]
$$

with a small time constant $\tau>0$.

Remark 16 It should be noted that the effects of some imperfections, such as small delays, sampling step, sampling noise, etc., always make it impossible to achieve the identity $e_{2}(t) \equiv 0$. In [28], Utkin succeeded in proving that if $e_{2}(t)$ remains within a $\delta$-vicinity of zero, e.g., $\left|e_{2}(t)\right| \leq \delta$, the output of the first-order filter (32) is close to the equivalent control $\Delta_{e q}(t)$ satisfying the following inequality

$$
\left|\bar{\Delta}_{e q}(t)-\Delta_{e q}(t)\right| \leq \mathcal{O}(\tau+\delta)+\mathcal{O}(\delta / \tau)
$$


From (33), it can be observed that estimation accuracy is of the order $\mathcal{O}(\tau)$ in an ideal case. When some imperfections are included, the estimation accuracy is of the order $\mathcal{O}(\max [\tau+\delta, \delta / \tau])$. In this case, in order to obtain a good estimation of $\Delta_{e q}(t)$, the filter time constant $\tau$ can be taken to be proportional to $\sqrt{\delta}$, i.e., $\tau=\sqrt{\delta}$, provided that $\delta$ is much smaller than 1. In this case, inequality (33) leads to $\left|\bar{\Delta}_{e q}(t)-\Delta_{e q}(t)\right| \leq \mathcal{O}(\sqrt{\delta})$. In addition, the constant $\tau$ should be selected to be larger than the sampling time of the computer-implemented filter.

A practical fixed-time output feedback control law for the perturbed double-integrator system is summarized in the following theorem.

Theorem 17 Consider system (25) with the output feedback control law

$$
\begin{aligned}
u(\hat{x}) & =-\left(k_{1}\left\lceil x_{1}\right\rfloor^{\varrho_{1}}+k_{1}^{\prime}\left\lceil x_{1}\right\rfloor+k_{1}^{\prime \prime}\left\lceil x_{1}\right\rfloor^{\varrho_{1}^{\prime}}\right)-\left(k_{2}\left\lceil\hat{x}_{2}\right\rfloor \varrho_{2}\right. \\
& \left.+k_{2}^{\prime}\left\lceil\hat{x}_{2}\right\rfloor+k_{2}^{\prime \prime}\left\lceil\hat{x}_{2}\right\rfloor^{\varrho_{2}^{\prime}}\right)-\bar{\Delta}_{e q}(t)
\end{aligned}
$$

where $\hat{x}_{2}$ is obtained from observer (11) with parameters given in (12), (27), (29) and $\bar{\Delta}_{e q}(t)$ is obtained through (32) with an appropriate time constant $\tau$. Then, the $o$ rigin of system (25) is practically fixed-time stable if the controller parameters $k_{i}, k_{i}^{\prime}, k_{i}^{\prime \prime}, \varrho_{i}$ and $\varrho_{i}^{\prime}(i=1,2)$ are $s$ elected as in Theorem 11.

Proof. Since the observer (11) with parameters in (12), (27) and (29) ensures that error dynamics in (28) is fixedtime stable, it follows that there exists a finite time, i.e., $T_{1}^{\prime}$, such that $\hat{x}_{1}=x_{1}, \hat{x}_{2}=x_{2}$ for all $t \geq T_{1}^{\prime}$. Next, we will show that the trajectory of system (25) under the output feedback control law (34) does not escape during finite time interval $\left[0, T_{1}^{\prime}\right]$. To this end, consider Lyapunov function (7). The derivative of $V$ can be calculated as

$$
\dot{V}=-\Pi x_{2}\left(k_{2}\left\lceil\hat{x}_{2}\right\rfloor^{\varrho_{2}}+k_{2}^{\prime}\left\lceil\hat{x}_{2}\right\rfloor+k_{2}^{\prime \prime}\left\lceil\hat{x}_{2} \varrho^{\varrho_{2}^{\prime}}+e_{\Delta}\right)\right.
$$

where $e_{\Delta}=\Delta(t)-\bar{\Delta}_{e q}(t)$ is bounded due to the boundness of $\Delta(t)$ and $\bar{\Delta}_{e q}(t)$. Following the same analysis for Cases 1 and 2 in the proof of Theorem 13, the following statements hold for the derivative of $V$.

Case 1: For $\left|x_{2}\right|>\left|e_{2}\right|$, implying $\operatorname{sign}\left(x_{2}-e_{2}\right)=$ $\operatorname{sign}\left(x_{2}\right), \dot{V}$ in (35) satisfies

$$
\begin{aligned}
\dot{V} & \leq \Pi\left|x_{2}\right|\left(\left|e_{\Delta}\right|-k_{2}\left|x_{2}-e_{2}\right|^{\varrho_{2}}-k_{2}^{\prime}\left|x_{2}-e_{2}\right|\right. \\
& \left.-k_{2}^{\prime \prime}\left|x_{2}-e_{2}\right|^{\varrho_{2}^{\prime}}\right)
\end{aligned}
$$

Case 2: For $\left|x_{2}\right| \leq\left|e_{2}\right|$, implying that $-x_{2}\left\lceil\left.\hat{x}_{2}\right|^{\vartheta} \leq\right.$ $\left|x_{2}\right|\left|x_{2}-e_{2}\right|^{\vartheta} \leq 2^{\vartheta}\left|e_{2}\right|^{\vartheta+1}$ holds for any $\vartheta>0, \dot{V}$ in (35) satisfies

$$
\begin{aligned}
\dot{V} & \leq \Pi\left(k_{2} 2^{\varrho_{2}}\left|e_{2}\right|^{\varrho_{2}+1}+2 k_{2}^{\prime}\left|e_{2}\right|^{2}+k_{2}^{\prime \prime} 2^{\varrho_{2}^{\prime}}\left|e_{2}\right|^{\varrho_{2}^{\prime}+1}\right. \\
& \left.+\left|e_{2}\right|\left|e_{\Delta}\right|\right)
\end{aligned}
$$

Since $e_{\Delta}$ and $e_{2}$ are bounded, it follows from (36) and (37) that $V$ and so $x_{1}$ as well as $x_{2}$ are bounded in the time interval $\left[0, T_{1}^{\prime}\right]$, which implies that $x_{1}\left(T_{1}^{\prime}\right)$ and $x_{2}\left(T_{1}^{\prime}\right)$ are finite values.

In what follows, we will discuss the trajectory evolution of system (25) under the control law (34) for all $t \geq T_{1}^{\prime}$.

Let $f_{u s}(\hat{x}, \delta)=\left[x_{2},-\left(k_{1}\left\lceil x_{1} \varrho_{1}^{\varrho_{1}}+k_{1}^{\prime}\left\lceil x_{1}\right\rfloor+k_{1}^{\prime \prime}\left\lceil x_{1}\right\rfloor \varrho_{1}^{\prime}\right)-\right.\right.$ $\left.\left(k_{2}\left\lceil\hat{x}_{2}\right\rfloor \varrho_{2}+k_{2}^{\prime}\left\lceil\hat{x}_{2}\right\rfloor+k_{2}^{\prime \prime}\left\lceil\hat{x}_{2}\right\rfloor \varrho_{2}^{\prime}\right)+e_{\Delta}\right]^{T}$ be the closedloop vector field of system (25) under the control law (34). Since $\hat{x}_{1}=x_{1}, \hat{x}_{2}=x_{2}$ for all $t \geq T_{1}^{\prime}$, it follows that $f_{u s}(\hat{x}, \delta)=f_{u s}(x, \delta)$ for any $t \geq T_{1}^{\prime}$. In this case, the closed-loop system can be rewritten as $\dot{x}=f_{u s}(x, \delta)$, which is homogeneous in the bilimit with associated triples $\left(\left(r_{u s_{0}}, \tau_{0}\right), k_{u s_{0}}, f_{u s_{0}}\right)$ and $\left(\left(r_{u s_{\infty}}, \tau_{\infty}\right), k_{u s_{\infty}}, f_{u s_{\infty}}\right)$, where $r_{u s_{0}}=\left[\frac{2-\varrho}{1-\varrho}, \frac{1}{1-\varrho}\right]^{T}, \tau_{0}=$ $\frac{\varrho}{1-\varrho}, k_{u s_{0}}=-1, f_{u s_{0}}=\left[x_{2},-k_{1}\left\lceil x_{1}\right\rfloor^{\varrho_{1}}-k_{2}\left\lceil x_{1}\right\rfloor^{\varrho_{2}}+\right.$ $\left.e_{\Delta}\right]^{T}$ and $r_{u s_{\infty}}=\left[\frac{2-\varrho}{1-\varrho}, \frac{3-2 \varrho}{1-\varrho}\right]^{T}, \tau_{\infty}=\frac{4-3 \varrho}{1-\varrho}, k_{u s_{\infty}}=$ $1, f_{u s_{\infty}}=\left[x_{2},-k_{1}^{\prime \prime}\left\lceil x_{1} \varrho^{\varrho_{1}}-k_{2}^{\prime \prime}\left\lceil x_{1} \varrho^{\varrho_{2}}+e_{\Delta}\right]^{T}\right.\right.$. Noting that $f_{u s}(x, 0)=f_{s}(x)$ and the system $\dot{x}=f_{s}(x)$ is fixed-time stable (see Theorem 11), it can be concluded that $\dot{x}=f_{u s}(x, 0)$ is fixed-time stable for all $t \geq T_{1}^{\prime}$. Therefore, it follows from Lemma 14 that there exists a continuous, positive definite and proper function $V(x)$ satisfying

$$
\frac{\partial V}{\partial x} f_{u s}(x, \delta) \leq-k_{V} \Gamma\left(V^{m}, V^{n}\right)+k_{\delta} \Gamma\left(\left|e_{\Delta}\right|^{p},\left|e_{\Delta}\right|^{q}\right)
$$

with $m=\frac{d_{V_{0}}+k_{u s_{0}}}{d_{V_{0}}}, n=\frac{d_{V_{\infty}}+k_{u s_{\infty}}}{d_{V_{\infty}}}, p=\frac{d_{V_{0}}+k_{u s_{0}}}{\tau_{0}}$ and $q=\frac{d_{V_{\infty}}+k_{u s_{\infty}}}{\tau_{\infty}}$. Since $k_{u s_{0}}=-1, k_{u s_{\infty}}=1$ and $d_{V_{0}}>$ $\max \left(\frac{2-\varrho}{1-\varrho}, \frac{1}{1-\varrho}\right)>2$ as well as $d_{V_{\infty}}>\max \left(\frac{2-\varrho}{1-\varrho}, \frac{3-2 \varrho}{1-\varrho}\right)>$ 3 (see Lemma 10) for any $\varrho \in(0,1)$, it follows that the conditions $0<m<1<n, p>0$ and $q>0$ hold. Since $\left|e_{\Delta}\right|<1$ can be satisfied after $t \geq T_{1}^{\prime}$ by selecting appropriate $\tau$, the conditions $\left|e_{\Delta}\right|^{q}<1$ and $\left|e_{\Delta}\right|^{p}<1$ hold. As a result, the term $\Gamma\left(\left|e_{\Delta}\right|^{p},\left|e_{\Delta}\right|^{q}\right)$ in (38) satisfies

$$
\Gamma\left(\left|e_{\Delta}\right|^{p},\left|e_{\Delta}\right|^{q}\right)=\frac{\left|e_{\Delta}\right|^{p}}{1+\left|e_{\Delta}\right|^{p}}\left(1+\left|e_{\Delta}\right|^{q}\right)<2\left|e_{\Delta}\right|^{p}
$$

after finite time $T_{1}^{\prime}$. Next, two different cases will be discussed. 
For $V \geq 1$, it follows that $\frac{1}{2}\left(1+V^{n}\right) \leq \Gamma\left(V^{m}, V^{n}\right)=$ $\frac{V^{m}}{1+V^{m}}\left(1+V^{n}\right)<\left(1+V^{n}\right)$ holds for $0<m<1<n$. Taking into account (39), (38) can be rewritten as

$$
\frac{\partial V}{\partial x} f_{u s}(x, \delta) \leq-\frac{k_{V}}{2}\left(1+V^{n}\right)+2 k_{\delta}\left|e_{\Delta}\right|^{p}, t \geq T_{1}^{\prime}
$$

Since $\left|e_{\Delta}\right|<1$ for $t \geq T_{1}^{\prime}$, condition $2 k_{\delta}\left|e_{\Delta}\right|^{p}<\frac{k_{V}}{2}$ can be satisfied by selecting appropriate $k_{V}$ and $k_{\delta}$. As a result, (40) can be rewritten as $\frac{\partial V}{\partial x} f(x, \delta) \leq-\frac{k_{V}}{2} V^{n}$. Integrating the inequality from $V=V_{0} \geq 1$ to $V=1$ obtains that the system trajectory reaches the level $V(x)=1$ in a finite-time $T_{2}^{\prime} \leq \frac{2\left(1-V_{0}^{1-n}\right)}{k_{V}(n-1)} \leq \frac{2}{k_{V}(n-1)}$ for $n>1$, which is independent of initial conditions.

For $V(x) \leq 1$, it follows from $0<m<1<n$ that $\frac{1}{2} V^{m} \leq \Gamma\left(V^{m}, V^{n}\right)=\frac{V^{m}}{1+V^{m}}\left(1+V^{n}\right) \leq V^{m}$. Taking into account (39), (38) can be rewritten as

$$
\frac{\partial V}{\partial x} f_{u s}(x, \delta) \leq-\frac{k_{V}}{2}(1-\theta) V^{m}+2 k_{\delta}\left|e_{\Delta}\right|^{p}-\frac{k_{V}}{2} \theta V^{m}
$$

for $t \geq T_{1}^{\prime}$, where $\theta \in(0,1)$. From (41), it follows that $\frac{\partial V}{\partial x} f_{u s}(x, \delta) \leq-\frac{k_{V}}{2}(1-\theta) V^{m}$ is strictly negative outside the set $\Xi=\left\{x \in \mathbb{R}^{2} \mid V(x) \leq V_{\epsilon}=\left[\frac{4 k_{\delta}\left|e_{\Delta}\right|^{p}}{k_{V} \theta}\right]^{1 / m}\right\}$. Integrating this inequality from $V=V_{0} \leq 1$ to $V=V_{\epsilon}$ obtains that the system trajectory reaches boundary $V=V_{\epsilon}$ in a finite-time $T_{3}^{\prime} \leq \frac{V_{0}^{1-m}-V_{\epsilon}{ }^{1-m}}{c(1-m)} \leq \frac{1-V_{\epsilon}^{1-m}}{c(1-m)} \leq$ $\frac{1}{c(1-m)}$ with $c=0.5 k_{V}(1-\theta)$, which is independent of initial conditions. Then, the system trajectory remains in the set $\Xi$ for all future time since $\frac{\partial V}{\partial x} f_{u s}(x, \delta)$ is strictly negative outside the set $\Xi$ and on the boundary of $\Xi$. The positive definiteness of $V(x)$ implies that all solutions of the perturbed double-integrator system (25) under the control law (34) converge to some bounded region when $V(x)$ belongs to the bounded closed set $\Xi$.

In summary, the solution of system (25) under the control law (34) converge into a bounded region in finitetime $T^{\prime} \leq T_{1}^{\prime}+T_{2}^{\prime}+T_{3}^{\prime}$ which is independent on initial conditions. Hence, the closed-loop system is practically fixed-time stable. This completes the proof.

Remark 18 It is worth noting that some classic algorithms, such as twisting [26], super-twisting algorithms [29] and their modified versions in [11,15] can be recovered formally from our proposed algorithms (3) and (11) with an appropriate selection of parameters, e.g., the algorithm in [15] is obtained from (11) if the parameter$s$ are specified as (27). However, the proof given in the paper is not applicable for the aforementioned classic algorithms due to the fact that the homogeneity in bi-limit requires the continuity of the right-hand sides.

\section{Simulation Example}

To confirm the property of fixed-time convergence with respect to the initial conditions, several different system initial values $x_{0}=[1,-1], x_{0}=\left[10^{2},-10^{2}\right]$ and $x_{0}=$ $\left[10^{4},-10^{4}\right]$ are considered. The simulation is carried out using Euler method with fixed sampling $10^{-4}$ second. To begin with, the fixed-time state feedback control law (3) with $k_{1}=k_{2}=2.0, k_{1}^{\prime}=k_{2}^{\prime}=0.6, k_{1}^{\prime \prime}=k_{2}^{\prime \prime}=3.0$ and $\varrho=0.6$ is applied. In this case, the simulation result is shown in Fig.1. From the sub-plot in Fig.1, it can be observed that the convergence time of the system subject to different initial values (including the one far away from the origin) is close, which shows a 'weak' dependence of the convergence time on the initial conditions. Then, the fixed-time output feedback control law (19) with observer (11) is tested. Without re-tuning the control parameters, the observer parameters are selected as $\bar{k}_{1}=\bar{k}_{2}=2.0, \bar{k}_{1}^{\prime}=\bar{k}_{2}^{\prime}=0.6, \bar{k}_{1}^{\prime \prime}=\bar{k}_{2}^{\prime \prime}=3.0$ and $\bar{\varrho}=0.6$. The initial value of the observer is set as $\hat{x}_{0}=[10,-10]$. In this case, the simulation result is shown in Fig.2. The rapid convergence under different initial conditions can also be observed when only output is available.

To examine the robustness of the method, an external disturbance $\Delta(t)=\frac{1}{2}[\sin (t)+\cos (t)]$ is added to the double integrator system (25). The observer (11) with parameters in (12) and (27) specified with $\bar{k}_{1}=1$ and $k_{2}=2.5$ is used. The initial values of the observer and the controller parameters are the same as those provided in the previous. The time constant $\tau=0.01$ in (32) is used in the simulation. In this case, the simulation results are plotted in Figs.3 and 4. From Fig.3, it can be seen that the system states converge to a small region around the origin quickly, which implies the practical fixed-time stability. Furthermore, Fig.4 shows that the external disturbances can be approximated in a finite time through equivalent control method.

Remark 19 Although the operation region of a plant is always bounded in practice, the fixed-time approach helps to design a control law, which is able to preserve the convergence time even with the variation of operation region. This property is especially useful in some cases where the bounded initial conditions are not known a priori. Some typical applications include state estimation, disturbance estimation and fault estimation, where the initial estimation error is bounded but unknown in practice. In these cases, it is difficult to estimate the convergence time using the traditional finite-time control methods, because the convergence time of the methods is heavily dependen$t$ on initial estimation errors. Another application is for the observation of a switched and hybrid systems with a positive dwell time [32,20]. In this case, one cannot guarantee the convergence of state estimation before the next plant's takes place if a bound for the initial conditions is 


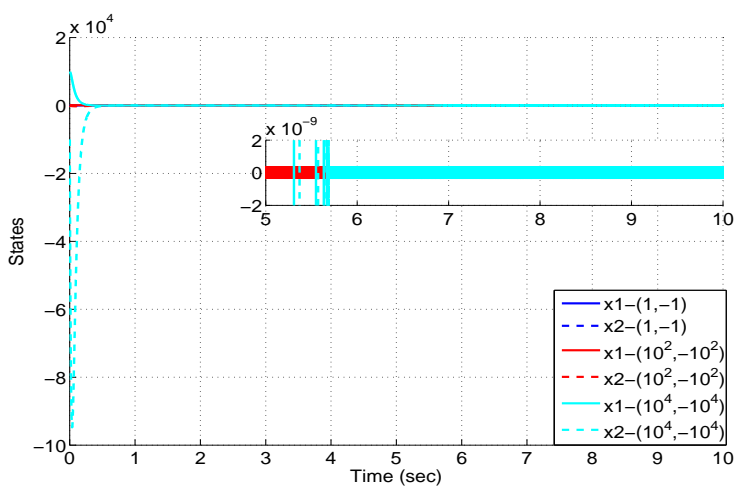

Fig. 1. The system responses by state feedback

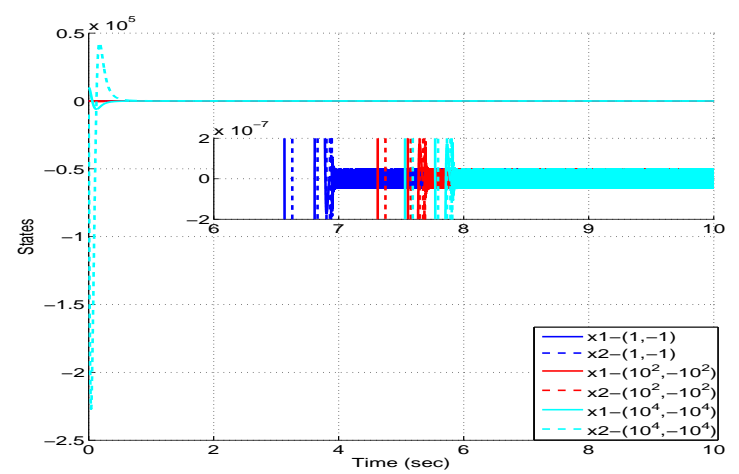

Fig. 2. The system responses by output feedback for nominal model

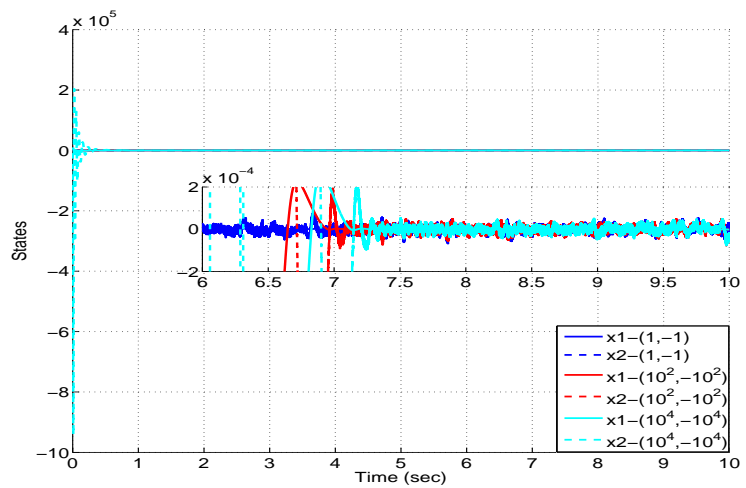

Fig. 3. The system responses by output feedback for perturbed model

not known a prior. Nevertheless, the fixed-time stabilization control can avoid these issues mentioned above.

\section{Conclusions}

A new fixed-time controller and observer are proposed and are then synthesized to present an unified framework for the fixed-time stabilization of a double-integrator system by output feedback. The results can be considered as an extension of the finite-time control algorith-

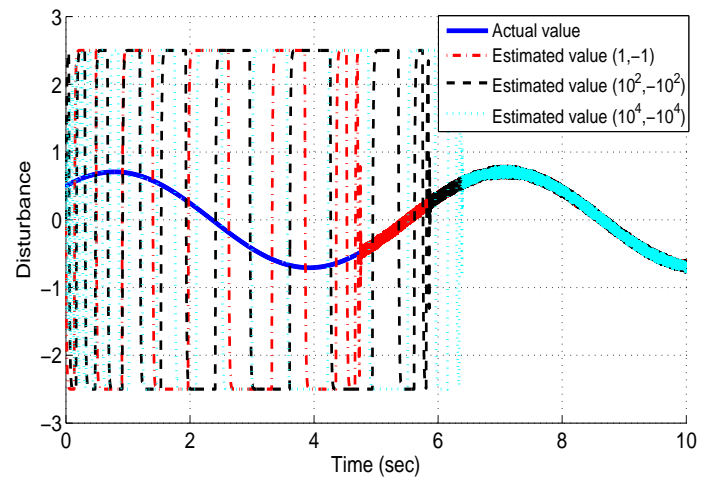

Fig. 4. The disturbance estimates

m. In our design, the higher order terms are added to achieve uniform convergence with respect to initial conditions and the lower order terms for the exact convergence in finite time. As a result, the property of fixedtime convergence is established through the combination of higher and lower order components. The homogeneity and the Lyapunov function are utilized to guarantee the fixed-time stability. Future work will focus on the extension of the results to higher order systems.

\section{References}

[1] Tian, B., Fan, W., Zong, Q., Wang, J., \& Wang, F. (2013). Non-singular terminal sliding mode control of rigid manipulators. Journal of the Franklin Institute, 350(7), 17871870.

[2] Gustafsson, T. (1996). On the design and implementation of a rotary controller. European Journal of Control, 2(3), 166175 .

[3] Capisani,L.M., Ferrara, A. \& Fridman, L.M. (2012). Manipulator fault diagnosis via higher order sliding-mode observers. IEEE Transaction on Industrial Electronics, 59(10), 3979-3986.

[4] Bhat, S.P., \& Bernstein, D.S. (2000). Finite-time stability of continuous autonomous systems. SIAM Journal of Control and Optimization, 38(3), 751-766.

[5] Polyakov, A., \& Poznyak, A. (2009). Lyapunov function design for finite-time convergence analysis: twisting controller for second-order sliding mode realization. Automatica, 45(2), 444-448.

[6] Lu, K.F., \& Xia, Y.Q. (2013). Adaptive attitude tracking control for rigid spacecraft with finite-time convergence. Automatica, 49(12), 3591-3599.

[7] Levant, A. (2005). Homogeneity approach to high order sliding mode design. Automatica, 41(5), 823-830.

[8] Levant, A. (2005). Quasi-continuous high-order sliding mode controllers. IEEE Transaction on Automatica Control, 50(11), 1812-1816.

[9] Pukdeboon, C., Zinober, A.S.I. \& Thein, M.W.L. (2010). Quasi-continuous higher order sliding-mode controllers for spacecraft-attitude-tracking maneuvers. IEEE Transaction on Automatica Control, 57(4), 1436-1444.

[10] Colbia-Vega, A., DeLeon-Morales, J. \& Fridman, L. (2008). Robust excitation control design using sliding-mode 
technique for multimachine power systems. Electric Power Systems Research, 78(9), 1627-1634.

[11] Orlov, Y., Aoustin, Y. \& Chevallereau, C. (2011). Finite Time Stabilization of a Perturbed Double Integrator-Part I: Continuous Sliding Mode-Based Output Feedback Synthesis. IEEE Transaction on Automatic Control, 56(3), 614-618.

[12] Andrieu, V., Praly, L., \& Astolfi, A. (2008). Homogeneous approximation, recursive observer design and Output Feedback. SIAM Journal on Control and Optimization, 47(4), 1814-1850.

[13] Polyakov, A. (2012). Nonlinear feedback design for fixed-time stabilization of linear control systems. IEEE Transactions on Automatic Control, 57(8), 2106-2110.

[14] Polyakov, A., Efimov,D., \& Perruquetti, W. (2015). Finitetime and fixed-time stabilization: Implicit Lyapunov function approach. Automatica, 51(2), 332-340.

[15] Cruz-Zavala, E., Moreno, J.A., \& and Fridman, L.M. (2011). Uniform robust exact differentiator. IEEE Transactions on Automatic Control, 56(11), 2727-2733.

[16] Zuo, Z., (2015). Non-singular Fixed-time Consensus Tracking for Second-order Multi-agent Networks. Automatica, 54(4), 305-309.

[17] Zuo, Z., (2016). Distributed robust finite-time nonlinear consensus protocols for multi-agent systems. International Journal of Systems Science, 47(6), 1366-1375.

[18] Zuo, Z., \& Tie, L. (2014). A new class of finite-time nonlinear consensus protocols for multi-agent systems. International Journal of Control, 87(2), 363-370.

[19] Parsegov.S., Polyakov.A., \& Shcherbakov, P. (2012). Nonlinear fixed-time control protocol for uniform allocation of agents on a segment. IEEE Conference on Decision and Control, 7732-7737, Hawaii, USA.

[20] Angulo, M.T., Moreno, J.A., \& Fridman, L. (2013). Robust exact uniformly convergent arbitrary order differentiator. Automatica, 49(8), 2489-2495.

[21] Filippov, A.F.

(1988)

Differential Equations with Discontinuous Right Hand Sides, Kluwer Academic Publishers, Dordrecht, The Netherlands, 1988.

[22] Polyakov, A., Fridman, L. (2014) Stability notions and Lyapunov functions for sliding mode control systems, Journal of the Franklin Institute 351, 1831-1865.

[23] Bacciotti, A. \& Rosier, L. (2005). Lyapunov Functions and Stability in Control Theory. Springer.

[24] Levant, A. (2013). On fixed and finite time stability in sliding mode control. 52nd IEEE Conference on Decision and Control, 4260-4265, Florence, Italy.

[25] Khalil, H.K. (2005). Nonlinear systems (3rd ed.). Upper Saddle River, NJ: Prentice Hall.

[26] Levant, A. (1993). Sliding order and sliding accuracy in sliding mode control. Internation Journal of control, 58(6), 1247-1263.

[27] Lunze, J., \& Lamnabhi-Lagarrigue, F. (2009). Handbook of hybrid systems control theory, tools, applications. Cambridge University Press.

[28] Utkin, V. (1992). Sliding Modes in Control and Optimization. Springer-Verlag, 1992.

[29] Davila, J., Fridman, L., \& Levant, A. (2005). Second order sliding mode observer for mechanical system. IEEE Transaction on Automatic Control, 50(11), 1785-1789.
[30] Chalanga, A., \& Bandyopadhyay, B. (2005). Output Stabilization of the Systems With Mismatched Uncertainties Using Higher Order Sliding Mode Approach. In the Proceedings of 10th Asian Control Conference, 23012306, Malaysia.

[31] Levant, A. (2003). Higher-order sliding modes, differentiation and outputfeedback control. International Journal of Control, 76(7-10), 924-941.

[32] Lunze, J., \& Lamnabhi-Lagarrigue, F. (2009). Handbook of hybrid systems control theory, tools, applications. Cambridge University Press.

[33] Utkin, V., \& Poznyak, A. (2013). Adaptive sliding mode control with application to super-twist algorithm: Equivalent control method. Automatica, 49(1), 39-47. 